\title{
Immune Modulation and Cytomegalovirus Reactivation in Sepsis-induced Immunosuppression: A Pilot Study
}

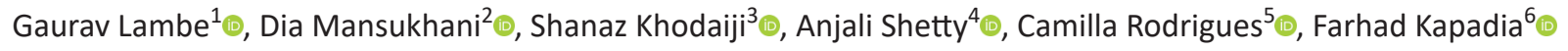

\begin{abstract}
Introduction: Sepsis is a life-threatening condition caused due to dysregulated immune response to an infection and progressive immunosuppression. Reactivation of cytomegalovirus (CMV) occurs frequently in sepsis and is found associated with adverse outcomes. The study objective was to evaluate the association between incidence of CMV reactivation and immune alteration in sepsis-induced immunosuppression in patients with prolonged sepsis.

Patients and methods: Patients admitted to intensive care unit (ICU), with severe sepsis and CMV immunoglobulin G (IgG) seropositivity, were prospectively enrolled. Other manifest immune suppression causes were excluded. Samples were collected on enrolment and further once a week until day 21 or death/discharge. CMV viral load was quantified using qPCR. Lymphocyte subset analysis (CD3+, CD4+, CD8+, CD19+, CD16+/CD56+, and CD25+CD127- regulatory T cells), human leukocyte antigen-DR isotype (HLA-DR) expression on monocytes, programmed death-1 (PD-1) expression on T lymphocytes, and proinflammatory (interleukin-6 (IL-6), tumor necrosis factoralpha (TNF- $\alpha$ ), and interferon-gamma (IFN- $\gamma$ )), anti-inflammatory cytokines levels (IL-2, IL-4, and IL-10) were analyzed by flow cytometry as markers for immunosuppression.

Results: A total of 25 CMV IgG-positive patients and 11 healthy controls were included. CMV reactivation occurred in 20 patients. Patients with CMV reactivation had T-cell lymphopenia. PD-1 expression on CD4+ and CD8+ T cells was markedly elevated $(p<0.02)$ in CMV-reactivated patients compared to nonreactivated patients. HLA-DR expression was significantly low on monocytes in all septic patients $(p<0.01)$ compared to healthy controls. IL-6 levels showed elevation at day 7, whereas IL-10 was found to be significantly higher from day 0 in CMV-reactivated group. Conclusion: Our study concluded that immune suppression markers and cytokine levels in patients with severe sepsis were found to be significantly associated with the incidence of $\mathrm{CMV}$ reactivation.

Keywords: Cytomegalovirus reactivation, Interleukin-10, Interleukin-6, Immunosuppression, Programmed death-1, Sepsis, Tregs.

Indian Journal of Critical Care Medicine (2022): 10.5005/jp-journals-10071-24079
\end{abstract}

\section{INTRODUCTION}

Sepsis is a life-threatening condition, which is recently defined as dysregulated host immune response to an infection due to impairment in innate and adaptive immunity. In 2017, the World Health Organization (WHO) has declared sepsis as a global health priority. ${ }^{1,2}$ Pathogen virulence factors and host immunity play a major role in sepsis-induced immune response and subsequent immune paralysis. Host's inability to recover from impaired immune function and progressive immune suppression despite clearance of infection by antibiotics plays a central role in prolonged or recurrent sepsis. ${ }^{3,4}$ Altered adaptive and innate immunity leads to major abnormalities in host response that results in an unresponsive immune state and increased susceptibility to secondary infections and reactivation of latent viruses.

Reportedly, numerous overlapping mechanisms in host immune responses contribute to protracted immunosuppression in sepsis. ${ }^{5-7}$ Effect of such immunosuppression on innate and adaptive immunity is evident with decreased human leukocyte antigen-DR isotype (HLA-DR) expression on antigen-presenting cells such as monocytes resulting in decreased antigen stimulation and cytokine production, ${ }^{8,9}$ persistent lymphopenia, and increased expression of coinhibitory programmed death-1 (PD-1) marker on CD4+ and CD8+ T lymphocytes, leading to cellular anergy and exhaustion, impaired cytokine production and elevated levels of regulatory T cells (Tregs). ${ }^{10-12}$

Increased immune tolerance (anergy) to antigen stimulation and suppressed immune functions has contributed to increased
'Department of Laboratory Medicine, PD Hinduja Hospital and Medical Research Centre, Mumbai, Maharashtra, India; Department of Critical Care, PD Hinduja Hospital and Medical Research Centre, Mumbai, Maharashtra, India

${ }^{2-5}$ Department of Laboratory Medicine, PD Hinduja Hospital and Medical Research Centre, Mumbai, Maharashtra, India

${ }^{6}$ Department of Intensive Care, PD Hinduja Hospital and Medical Research Centre, Mumbai, Maharashtra, India

Corresponding Author: Shanaz Khodaiji, Department of Laboratory Medicine, PD Hinduja Hospital and Medical Research Centre, Mumbai, Maharashtra, India, Phone: +91 9820551848, e-mail: skhodaiji@ hotmail.com

How to cite this article: Lambe G, Mansukhani D, Khodaiji S, Shetty A, Rodrigues C, Kapadia F. Immune Modulation and Cytomegalovirus Reactivation in Sepsis-induced Immunosuppression: A Pilot Study. Indian J Crit Care Med 2022;26(1):53-61.

Source of support: Nil

Conflict of interest: None

incidence of nosocomial infections and reactivation of latent viruses such as cytomegalovirus (CMV) in prolonged sepsis. ${ }^{13-16}$ Though many studies have provided insights into mechanisms contributing to immune suppression in sepsis, there is a paucity of significant data highlighting the association of sepsis-induced immune suppression and the incidence of CMV reactivation in prolonged sepsis. 
Given the above consideration, the objectives of the prospective, observational study were (1) to evaluate the incidence of CMV reactivation in a group of patients with prolonged sepsis as reflected by length of stay (LOS) and (2) to evaluate the association of CMV reactivation in sepsis with immune markers such as lymphocyte subsets, PD-1 expression on T lymphocytes, HLA-DR expression on monocytes, and serum cytokine levels determined by flow cytometry.

\section{Materials and Methods}

This prospective, observational study was conducted at a tertiary care hospital. The study was approved by the Institutional Review Board (IRB) of the hospital. Consecutive patients admitted or transferred to intensive care unit (ICU) from within the hospital with severe sepsis were daily screened for the selection criteria. Patients were recruited after written informed consent was obtained from the patients or their next of kin.

\section{Inclusion Criteria}

The inclusion criteria concern critically ill adult patients with severe sepsis (1) with more than 7-14 days anticipated LOS in the ICU and CMV IgG seropositive.

\section{Exclusion Criteria}

The exclusion criteria concern the patients who were less than 18 years old, CMV IgG seronegative, or on anti-CMV treatment for 7 days prior to enrolment in the study, those with hematological or immunological disease, who were receiving chemotherapy, radiation, transplant drugs, or immunosuppressive treatment in the last 1 month or who had a manifestation of immunosuppression due to HIV or other diseases.

Among the enrolled patients, severity of illness on admission was measured by Sequential [sepsis-related] Organ Failure Assessment (SOFA) scoring system. ${ }^{17}$

\section{Study Design}

Patients fulfilling the selection criteria were enrolled in the study. Enumeration of lymphocyte subsets (T, B, and NK cells), Tregs, and expression of HLA-DR on monocytes and expression of PD-1 on T lymphocytes (CD4+, CD8+) was performed on the flow cytometer. A control group of eleven healthy volunteers (HVs) with no abnormal laboratory findings and comorbidities was also analyzed for the above-mentioned immune parameters.

\section{Blood Sampling}

Peripheral blood samples were obtained from patients admitted to the ICU on enrolment (day 0) and once a week thereafter for 3 weeks (days 7, 14, and 21), or until ICU discharge or death. Two to three milliliters of whole blood was collected in two ethylenediaminetetraacetic acid (EDTA) tubes and one plain serum vacutainer. One EDTA tube was used for flow cytometric analysis. The other EDTA tube was centrifuged at $500 \mathrm{~g}$ for 5 minutes at $4^{\circ} \mathrm{C}$ to obtain plasma, which was transferred in aliquots and stored at $-80^{\circ} \mathrm{C}$ until further analysis. Serum tube was kept at room temperature for 1 hour for clotting process and then centrifuged at $500 \mathrm{~g}$ for 5 minutes at room temperature to obtain serum. Aliquots were prepared and stored at $-80^{\circ} \mathrm{C}$ until assays were performed.

\section{Serology}

CMV IgG was performed as a screening test, prior to the patient's enrolment. This was performed using CMV IgG enzyme-linked immunoassay (ELISA) (Dia.Pro Diagnostic Bioprobes, Milano, Italy). Only seropositive patients were included in the study.

\section{Flow Cytometry for Immune Markers}

Characterization of lymphocyte subsets was performed using Multitest 6-color TBNK reagent containing CD3 FITC/CD16 $\mathrm{PE}+$ CD56 PE/CD45 PerCP-Cy5.5/CD4 PE-Cy7/CD19 APC/CD8 APC-Cy7 (BD Biosciences).

Analysis of PD-1 expression on T lymphocytes was performed using Brilliant Violet (BV510)-conjugated anti-PD-1 antibody (clone EH12.1 from BD Biosciences). Expression of PD-1 was measured as median fluorescence intensity (MFI) on CD3+ T lymphocyte population, derived from CD45+ lymphocyte population.

Expression of HLA-DR on monocytes was assessed using Brilliant Violet (BV421)-conjugated anti-HLA-DR (BD Biosciences) antibody (clone G46-6). HLA-DR expression was measured as MFI on the monocyte population obtained by gating on CD45 vs side scatter (SSC) population.

For characterization of Tregs subset, EDTA whole blood was stained with Human Regulatory T cell cocktail reagent [FITC antiHuman CD4/PE-Cy7 anti-Human CD25/Alexa Fluor 647 anti-Human CD127 (BD Biosciences)].

Stain-lyse-wash protocol was adopted for all flow cytometry assays mentioned above.

\section{Measurement of Proinflammatory and Anti- inflammatory Cytokines}

For quantitative analysis of proinflammatory (IL-2, IL6, TNF- $\alpha$, and IFN- $\gamma$ ), anti-inflammatory (IL-4 and IL-10), and IL-17 cytokines, $50 \mu \mathrm{L}$ of patient's serum was reacted with $50 \mu \mathrm{L}$ of conjugated beads. Samples were processed according to the manufacturer's instructions. Mixed capture beads $(50 \mu \mathrm{L})$ and each serum sample $(50 \mu \mathrm{L})$ were incubated for 1 hour at room temperature and then phycoerythrin (PE)-tagged detection reagents $(50 \mu \mathrm{L})$ were added to the bead-sample mixture and incubated for 2 hours at room temperature. The samples were washed and analyzed on BD FACS Canto II Flow Cytometer (BD Biosciences). Data analysis was carried out with FCAP Array software, version 3.0 for Windows.

\section{Quantification Assay}

CMV DNA was quantified in stored plasma samples using RealStar CMV qPCR Kit [Altona Diagnostic Technologies (ADT), Hamburg, Germany]. Viral DNA was extracted and eluted in an elution buffer. The assay was performed on ABI 7500 Fast Dx Real-time PCR system. PCR conditions were processed according to the manufacturer's instructions. CMV reactivation was measured as CMV DNA copies and categorized into two groups, less than 1000 copies $/ \mathrm{mL}$ and greater than 1000 copies $/ \mathrm{mL}^{18}$

\section{Statistical Analysis}

Statistical analysis was performed using R software V3.5.1. Results for continuous variables were reported as median and interquartile (IQ) range. Results for qualitative variables were reported as frequency and percentages. Shapiro-Wilk test was used to determine whether data sets differed from a normal distribution.

Results for quantitative variables were reported as mean and standard deviation ( \pm SD). Intragroup (>1000 copies $/ \mathrm{mL}$, 
$<1000$ copies/mL, and CMV nonreactive group) was compared using one-way analysis of variance (ANOVA) for each time point. Post hoc analysis was performed using Kruskal-Wallis test. Paired $t$-test was used to analyze the results at defined time points.

\section{Results}

Twenty-five patients were selected for the study as per the study design (Flowchart 1).

CMV-reactivated and nonreactivated patients showed almost similar severity of illness based on SOFA scoring. The clinical characteristics of patients with sepsis and healthy controls are described in Table 1.

\section{Incidence of CMV Reactivation}

CMV reactivation occurred in 20 out of 25 sepsis patients (80\%). Median time point for viral reactivation was 7 days (0-21 days). CMV reactivation with $>1000$ copies $/ \mathrm{mL}$ was observed in 12 patients (group I) and $<1000$ copies $/ \mathrm{mL}$ in 8 patients (group II). Absence of CMV reactivation was observed in five patients (group III). In our study, eight patients died and four patients survived in the group of CMV reactivation $>1000$ copies $/ \mathrm{mL}$ (group I), whereas two patients died and six patients survived in the group of CMV reactivation $<1000$ copies/mL (group II).

The time at which CMV viremia was first detected in these patients is shown in Table 2.

\section{Result of Lymphocyte Subsets}

A significant decrease in T lymphocyte counts was observed in all patients with sepsis (groups I, II, and III) on the day of enrolment as compared to healthy controls $(638.39+503.831$ vs $1939.56+873.54$, $p=0.00002)$. Further, group I patients with CMV viremia $>1000$ copies/mL had T-cell lymphopenia as compared to group II

Flowchart 1: Schematic representation of study design

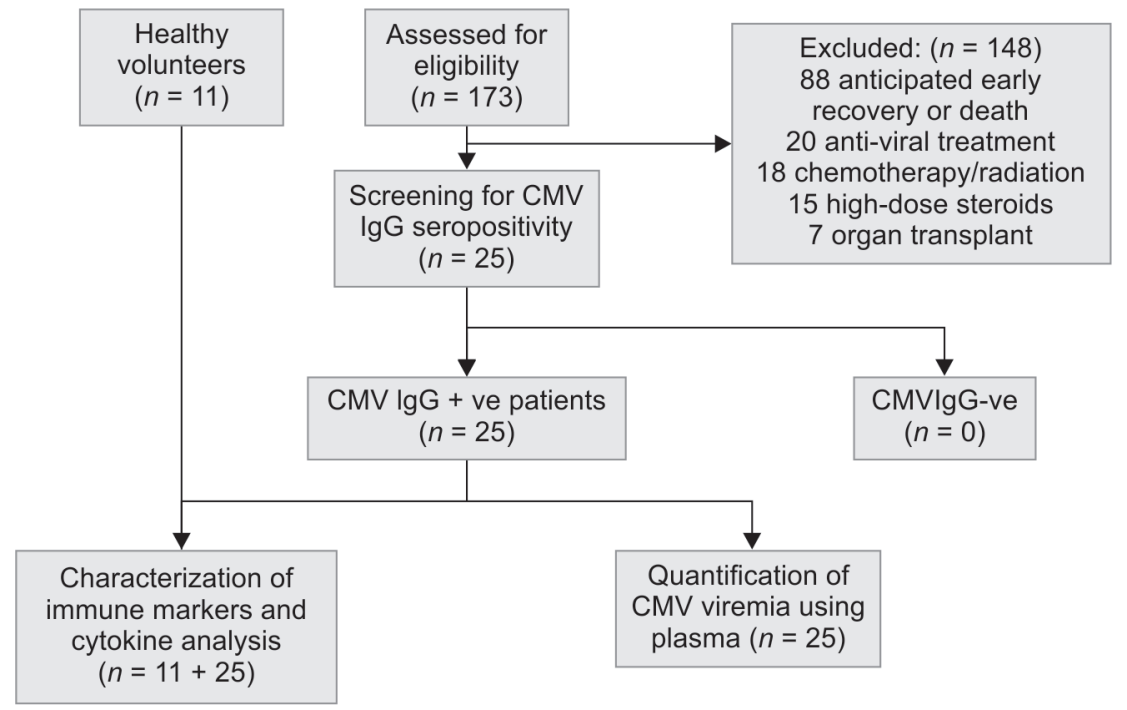

Table 1: Clinical characteristics of patients

\begin{tabular}{|c|c|c|c|c|c|}
\hline \multicolumn{6}{|c|}{ Patient characteristics } \\
\hline \multirow[b]{2}{*}{ Characteristics } & \multirow[b]{2}{*}{ All patients $(n=25)$} & \multicolumn{2}{|c|}{ CMV-positive $(n=20)$} & \multirow[b]{2}{*}{ CMV-negative $(n=5)$} & \multirow[b]{2}{*}{ Healthy control $(n=11)$} \\
\hline & & $C M V>1000(n=12)$ & $C M V<1000(n=8)$ & & \\
\hline Age (years) & $60(47-85)$ & $64(46-76)$ & $60.5(33-85)$ & $57(42-70)$ & $54(30-69)$ \\
\hline Gender, M:F & $17: 8$ & $9: 3$ & $4: 4$ & $4: 1$ & $6: 5$ \\
\hline SOFA score & $8(1-15)$ & $9(5-15)$ & $4.5(1-9)$ & $8(3-12)$ & NA \\
\hline \multicolumn{6}{|l|}{ Primary diagnosis } \\
\hline Medical & 18 & 10 & 6 & 2 & NA \\
\hline Surgical & 7 & 2 & 2 & 3 & NA \\
\hline \multicolumn{6}{|l|}{ Source of infection } \\
\hline Respiratory & 6 & 2 & 3 & 1 & NA \\
\hline Urinary & 4 & 3 & 1 & 0 & NA \\
\hline Blood & 10 & 5 & 3 & 2 & NA \\
\hline Abdomen & 1 & 0 & 1 & 0 & NA \\
\hline Cellulitis/soft tissue & 2 & 1 & 0 & 1 & NA \\
\hline Unidentified & 2 & 1 & 0 & 1 & NA \\
\hline
\end{tabular}

SOFA, sequential organ functional assessment; M:F, male:female ratio. Data are expressed as median [IQ range], number or ratio; NA, not applicable 
Table 2: Time for detection of CMV reactivation

\begin{tabular}{lcccc}
\hline Time & Day 7 & Day 14 & Day 21 & Total $(n)$ \\
\hline $\begin{array}{l}\text { No. of patients with CMV } \\
\text { reactivation }>1000 \text { copies } / \mathrm{mL}\end{array}$ & 10 & 2 & 0 & 12 \\
$\begin{array}{l}\text { No. of patients with CMV } \\
\text { reactivation }<1000 \text { copies } / \mathrm{mL}\end{array}$ & 4 & 3 & 1 & 8 \\
$\begin{array}{l}\text { Total no. of patients reactivated }(n) \\
\text { CMV, cytomegalovirus }\end{array}$ & 14 & 5 & 1 & 20 \\
\hline
\end{tabular}
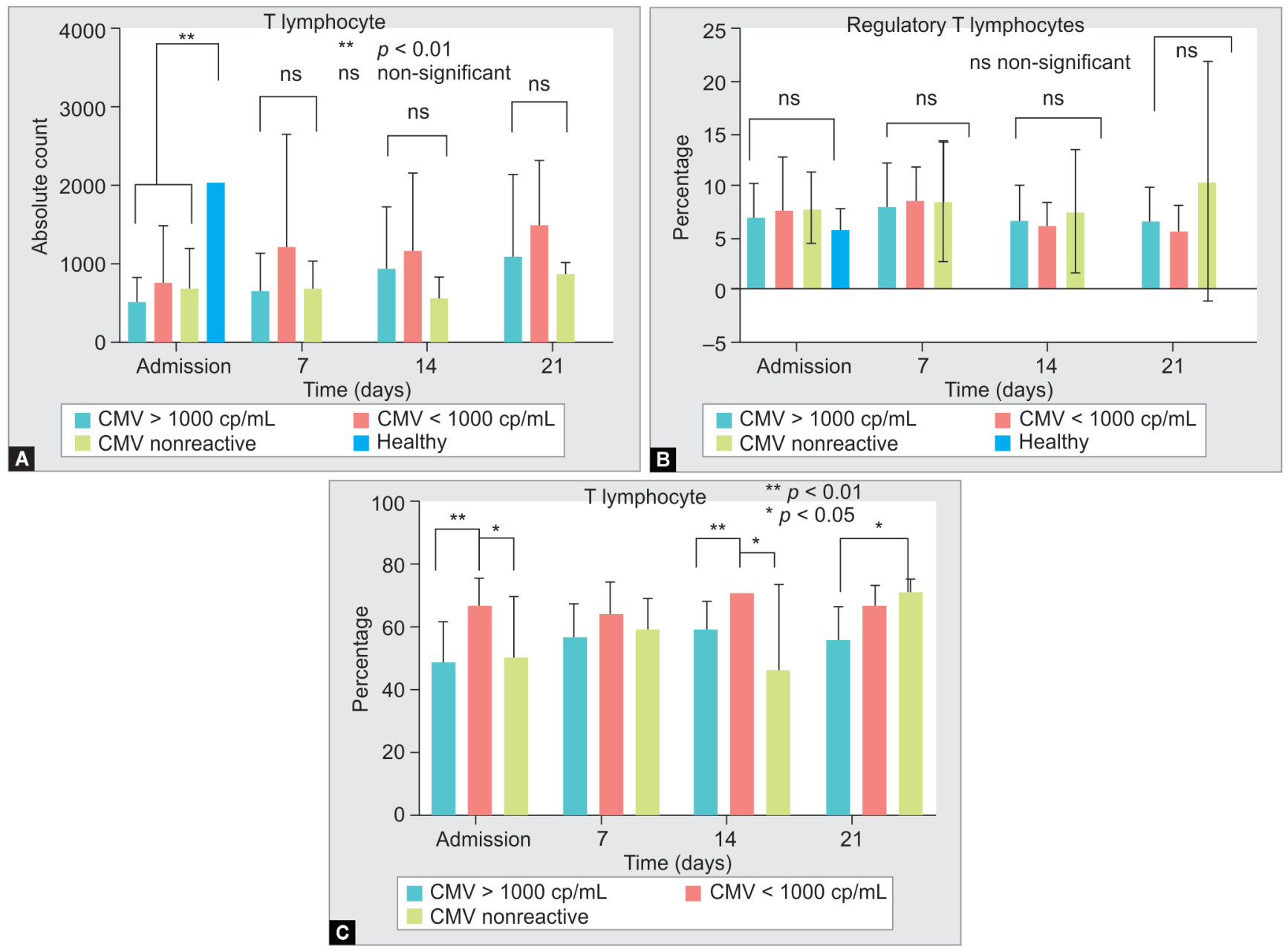

Figs $1 \mathrm{~A}$ to $\mathrm{C}$ : Lymphocyte subsets in the study population

$(521.04+316.60$ vs $777.11+718.61)$ and group III $(521.04+316.60$ vs $698.15+509.24)$ on day 0. T lymphocyte counts in CMV-reactivated patients, i.e., group I and group II, showed a gradual increase, whereas in group III, patients with no CMV reactivation remained low on day 14 and day 21. It is to be noted that no significant difference in T cell counts was observed between group III and groups I and II, except on the day of enrolment. We did not observe any significant changes in B lymphocyte counts and natural killer (NK) lymphocytes among the three groups. Lymphocyte subset counts in the study population with time points are shown in Figure 1.

\section{Tregs}

The percentage of Tregs in all three groups was found to be high compared to group IV, but no significant difference was observed between all three groups over the study period (Fig. 1).

\section{HLA-DR Expression on Monocytes}

In group IV, HLA-DR expression on monocytes was significantly higher $(p>0.0002)$ compared to all three patient groups as measured by the MFI, which was $7006.5 \pm 2469$ in sepsis patients vs $29902.18 \pm 10154$ in group IV (Fig. 2). It was observed that HLA-DR expression in patients of groups I and II increased with time as compared to group III patients. However, there was no significant difference between these groups (Fig. 2).

\section{PD-1 Expression on CD4+ and CD8+ T Lymphocyte Subset}

On day 0 , there was no overexpression of PD-1 on T lymphocyte subsets (CD4+, CD8+) in all three groups of patients, similar to that seen in group IV. In addition, no significant difference was 



Figs 2A to D: HLA-DR expression on monocyte population

observed in PD-1 expression on CD4+ $(p=0.72)$ and CD8+ T lymphocyte subsets $(p=0.78)$ between all three groups and healthy controls. However, we observed that PD-1 expression on CD4+ and CD8+ lymphocytes started to increase in groups I and II with time. On enrolment, PD-1 expression on CD4+ lymphocytes showed MFI of $304.16+191.58$ and $364.25+201.56$ in groups I and II, respectively, vs $342.20+82.78$ in group III. CD8+ lymphocytes showed an MFI of $386.91+332.34$ and $450.12+261.50$ in groups I and II, respectively, vs $337.40+204.02$ for group III. However, with prolonged stay, on day 7, expression of PD-1 on CD8+ T lymphocytes showed a significant increase $(p=0.02)$ in group I (MFI 515.91 + 526.86) and group II (MFI 580.12 + 246.52) compared to group III (MFI 174.20 +47.88). A similar pattern was seen on day $14(p=0.03)$ as the MFI for group I was $587.75+588.65$ and group II was $560.87+260.27$ vs group III in which MFI was $116.20+77.04$. Though PD-1 expression on CD4+ve T lymphocytes was found to increase after day 0 in group I and group II as compared to group III, this was not statistically significant. Group III patients showed a gradual decrease in PD-1 expression on CD4+ and CD8+ T lymphocytes during the study period (Fig. 3).

\section{Cytokines Levels}

Levels of IL-10 were found to be elevated in all patients with sepsis compared to healthy controls. On day $0, \mathrm{IL}-10$ levels were significantly higher $(p=0.037)$ in group I (18.71 \pm 30.86

$\mathrm{pg} / \mathrm{mL})$ compared to group II (8.54 $\pm 3.70 \mathrm{pg} / \mathrm{mL})$ and group III $(5.00 \pm 2.95 \mathrm{pg} / \mathrm{mL})$. Further, it was observed that these levels increased with time in group I, but did not increase significantly in other groups. Cytokine levels of all study groups over the period are shown in Figure 4.

IL-6 levels were found to be increase in group III with no CMV reactivation on day 0 , compared to those who developed CMV reactivation ( $171.77 \pm 146.72$ vs $118.33 \pm 76.37,127.41 \pm 123.74 \mathrm{pg} /$ $\mathrm{mL}, p=0.693$ ). However, with LOS IL-6 levels started to increase in group I and these levels remained high throughout the study period compared to levels in group II patients and group III patients (Fig. 4).

Levels of IFN- $\gamma$ showed a decreasing trend in group I compared to group III. In group II, levels started rising significantly $(p=0.01)$ over time as compared to group I $(6.20 \pm 2.46$ vs $2.59 \pm 1.96)$. However, group III showed no major difference in IFN- $\gamma$ levels over the study period (Fig. 4).

Similarly, levels of TNF- $\alpha$ and IL-2 were found to be elevated in group I on day 0 and increased on day 7 and day 14, after which they declined. There was no significant difference observed among the groups (Fig. 4).

\section{Discussion}

Sepsis remains the most common medical condition associated with high mortality and morbidity in ICUs. Sepsis-induced immunosuppression is known to be associated with early 

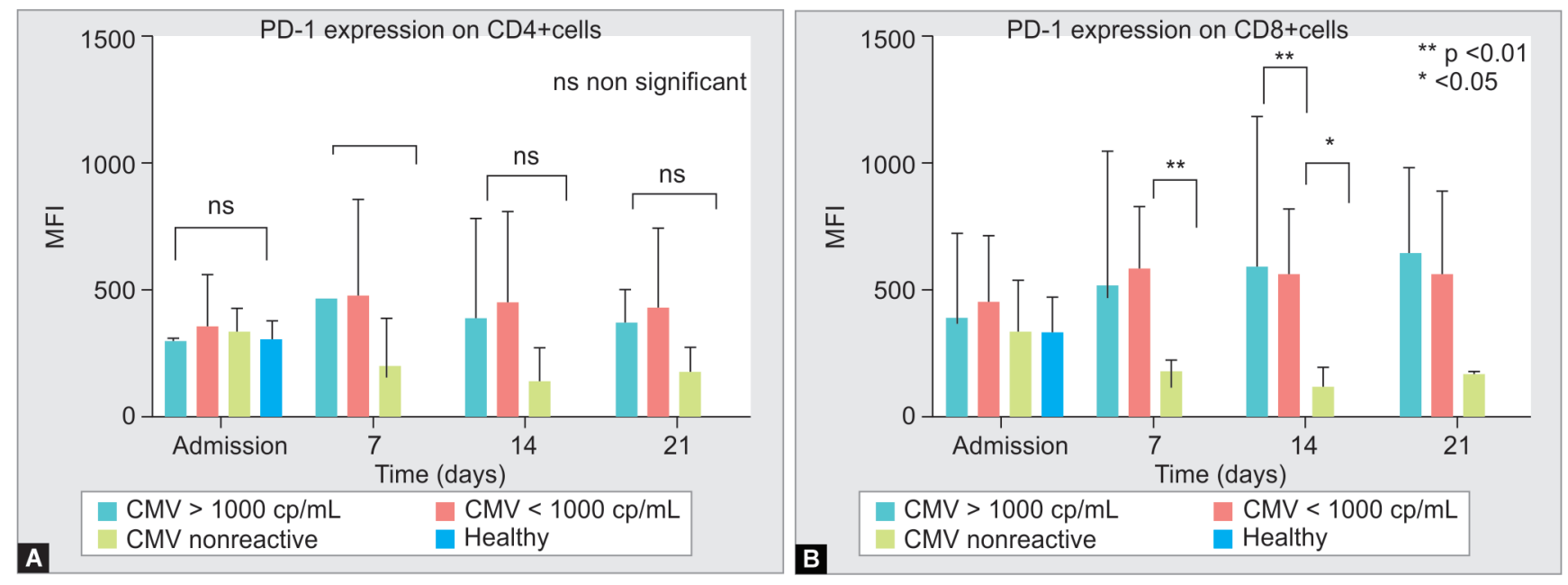

Figs 3A and B: PD- 1 expression on CD4+ and CD8+ T lymphocytes

inflammatory phase, followed by a compensatory immune response involving major modulations in the immune mechanism. This is demonstrated by markedly decreased HLA-DR expression on monocytes, increased expression of PD-1 on T lymphocytes resulting in anergy and loss of T-lymphocyte function. ${ }^{3,19}$ Significant lymphopenia and increased regulatory $T$ cell population are also correlated with suppressed immune state. ${ }^{7}$ Sepsis-induced immunosuppression has been identified as a risk factor for opportunistic nosocomial infections and reactivation of latent viruses such as CMV in immunocompetent patients. ${ }^{13,15}$

In this study, we demonstrated the incidence of CMV reactivation in a group of immunocompetent patients with severe sepsis admitted to the ICU. We observed $80 \%$ of the participants reactivated for CMV with increasing LOS. This finding is consistent with previous studies showing that CMV reactivation occurs frequently in critically ill adult patients with sepsis and associated with adverse outcomes. ${ }^{13,14}$ We found the median time for CMV reactivation in our sepsis group as 7 days. Similarly, Walton et al. and Tomino et al. observed CMV reactivation with the median of 7-13 days. ${ }^{13,15}$ However, Limaye et al. and other studies have observed a quantitative relationship between CMV viremia and prolonged hospital stay (death or 30 days) in critically ill patients with sepsis suggesting a strong rationale for early assessment of $\mathrm{CMV}$ viremia to prevent $\mathrm{CMV}$ reactivation and associated outcomes in sepsis. ${ }^{14,16}$

HLA-DR, expressed on monocytes primarily presents microbial antigen to lymphocytes for the activation of cellular and humoral response. ${ }^{19}$ Studies have reported a significant loss of HLA-DR expression on monocytes in patients with sepsis and septic shock as compared to healthy individuals. ${ }^{8,15}$ Similarly, we observed decreased HLA-DR expression on monocytes in patients with sepsis as compared to healthy controls. However, patients with CMV reactivation did not show a significant difference in HLA-DR expression compared to patients with no CMV reactivation. This indicates that HLA-DR expression on monocytes was not correlated to the incidence of CMV reactivation. However, it could be used as a surrogate marker of early sepsis immunosuppression as also observed by other investigators. ${ }^{20}$

Recent studies by Adrie et al. and Drewry et al. observed that lymphopenia on ICU admission and its persistence on 3rd to 4th day was associated with an increased risk of opportunistic infections in critically ill patients with severe sepsis. ${ }^{21-23}$ In the present study, we found significant and persistent T cell lymphopenia in patients who had CMV viremia greater than 1000 copies/mL. On the contrary, lymphopenia was less severe in patients with no CMV reactivation and it showed improvement after day 14.

The PD-1 inhibitory receptor regulates effector T-cell responses, T-cell tolerance, and T-cell exhaustion. ${ }^{14}$ In immunocompromised patients, upregulation of PD-1 expression on T lymphocytes has been correlated with CMV disease, whereas increased PD-1 expression was also linked to immunosuppression in sepsis studies. ${ }^{24,25}$ We observed that PD-1 on CD4+ve and CD8+ve T lymphocytes was significantly expressed and kept on increasing in patients with CMV reactivation with LOS. Patients with no CMV reactivation showed a decreasing trend in PD-1 expression on CD4+ and CD8+ T lymphocytes with time. This result is consistent with the findings of other studies, suggesting that PD-1 expression of $\mathrm{T}$ lymphocyte correlates with CMV reactivation in severe sepsis. ${ }^{15,26}$

IL-10 is a key component that regulates the potentially excessive immune responses during infection. Studies have reported that IL-10 cytokine levels were significantly high in patients with sepsis and this was associated with cytokine-mediated suppression of innate and adaptive immunity. ${ }^{27} \mathrm{CMV}$ induces the upregulation of hIL-10 in monocyte-derived cells, thereby amplifying IL-10mediated immunosuppression with suppressed monocytes and CD4+, CD8+ lymphocyte function and favoring the chronicity. ${ }^{28,29}$ In the current study, elevated levels of IL-10 cytokine were observed in patients with high CMV viral load $(>1000 \mathrm{cp} / \mathrm{mL})$, and the levels were significantly high compared to other groups $(<1000 \mathrm{cp} / \mathrm{mL}$ and nonreactivated) throughout the LOS. This has been consistent with other studies suggesting that IL-10 is associated with CMV reactivation as sepsis progresses and can be used as a marker of predicting mortality in sepsis. ${ }^{30}$

Role of IL- 6 has been demonstrated as a predictor of severity in sepsis along with C-reactive protein (CRP) and procalcitonin (PCT). Elevated levels of IL- 6 have been reported during acute hyperinflammatory phase in severe sepsis followed by compensatory immunosuppression. ${ }^{31,32}$ In context, CMV reactivation has been reported in highly inflammatory environments like transplant, cancer, and which correlates to sepsis due to the initial proinflammatory phase. ${ }^{33,34}$ In the present study, IL-6 levels were 



A



c
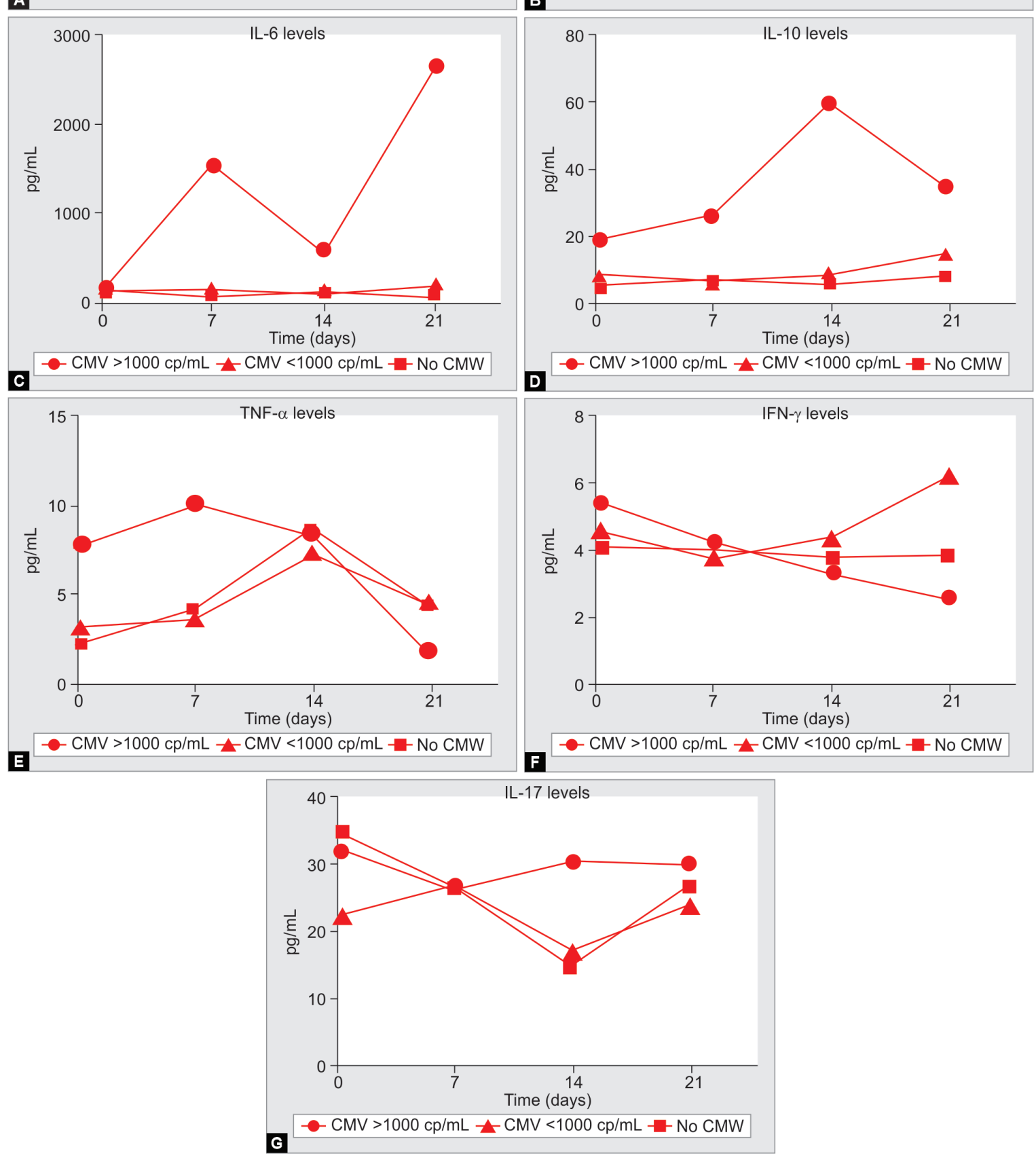

Figs 4A to G: Comparison of cytokine levels between three groups with time 
observed to be comparatively very high in patients with CMV reactivation and showed an increased trend with LOS in group I. In view of these observations, IL- 6 can be considered as an early marker for the prediction of CMV reactivation in sepsis patients.

The limitation of the study was that the sample size was small. We did not include age-matched controls and critically ill nonseptic patients.

\section{CONCLUSION}

Our study demonstrated the high prevalence of CMV reactivation in a cohort of immunocompetent patients with sepsis. The incidence of reactivation was found to be associated with sepsis-induced immune suppression markers like upregulation of PD-1 expression on CD4+ and CD8+ T lymphocytes and elevated levels of IL-6 and IL-10 cytokines. Monitoring of immune markers such as PD-1 expression and serum level of IL- 6 and IL-10 cytokines may play diagnostic role in predicting $\mathrm{CMV}$ reactivation in patients with prolonged sepsis.

\section{Author's Contribution}

The study conception and design were done by Gaurav Lambe, Dia Mansukhani, Shanaz Khodaiji, Anjali Shetty, Camilla Rodrigues, and Farhad Kapadia. Gaurav Lambe carried out the experimental work and data collection. The data analysis and interpretation were conducted by Gaurav Lambe, Camilla Rodrigues, Dia Mansukhani, Shanaz Khodaiji, and Farhad Kapadia. Gaurav Lambe, Dia Mansukhani, Shanaz Khodaiji, and Farhad Kapadia prepared the manuscript draft and reviewed the article.

\section{ORCID}

Gaurav Lambe 이 https://orcid.org/0000-0002-4640-1657 Dia Mansukhani (1) https://orcid.org/0000-0003-1787-1024 Shanaz Khodaiji @ https://orcid.org/0000-0001-7150-8889 Anjali Shetty @ 1 https://orcid.org/0000-0001-9575-4080 Camilla Rodrigues (10 https://orcid.org/0000-0002-6105-6660 Farhad Kapadia (1) https://orcid.org/0000-0003-1837-1144

\section{References}

1. Singer M, Deutschman CS, Seymour C, Shankar-Hari M, Annane $D$, Bauer $M$, et al. The third international consensus definitions for sepsis and septic shock (sepsis-3). JAMA-J Am Med Assoc 2016;315(8): 801-810. DOI: 10.1001/jama.2016.0287.

2. Goodman CW, Brett AS. Gabapentin and pregabalin for pain - is increased prescribing a cause for concern? N Engl J Med 2017; 377(5):411-414. DOI: 10.1056/NEJMp1704633.

3. Lewis DH, Chan DL, Pinheiro D, Garden OA. Immunopathology of sepsis: review article. J Vet Intern Med 2012;457-482. DOI: 10.1111/j.1939-1676.2012.00905.x.

4. Boomer JS, To K, Chang KC, Takasu O, Osborne DF, Walton AH, et al. Immunosuppression in patients who die of sepsis and multiple organ failure. JAMA-J Am Med Assoc 2011;306(23):2594-2605. DOI: 10.1001/ jama.2011.1829.

5. Muenzer JT, Davis CG, Chang K, Schmidt RE, Dunne WM, Coopersmith $\mathrm{CM}$, et al. Characterization and modulation of the immunosuppressive phase of sepsis. Infect Immun 2010;78(4):1582-1592. DOI: 10.1128/ IAI.01213-09.

6. Iskander KN, Osuchowski MF, Stearns-Kurosawa DJ, Kurosawa S, Stepien D, Valentine C, et al. Sepsis: multiple abnormalities, heterogeneous responses, and evolving understanding. Physiol Rev 2013;93(3):1247-1288. DOI: 10.1152/physrev.00037.2012.

7. Muszynski JA, Hall MW. Sepsis-induced innate and adaptive immune suppression. Open Inflamm J 2011;4(1):67-73.
8. Winkler MS, Rissiek A, Priefler M, Schwedhelm E, Robbe L, Bauer A et al. Human leukocyte antigen (HLA-DR) gene expression is reduced in sepsis and correlates with impaired TNF $\alpha$ response : a diagnostic tool for immunosuppression? PLoS One 2017;12(8):e0182427. DOI: 10.1371/journal.pone.0182427.

9. Wang J, Li L, Shi S. Expression of monocyte HLA-DR and blood lactic acid level in patients with sepsis and correlation with prognosis. Int J Clin Exp Med 2018;11(5):4905-4911.

10. Yi JS, Cox MA, Zajac AJ. T-cell exhaustion: characteristics, causes and conversion. Immunology 2010;129(4):474-481. DOI: 10.1111/j.13652567.2010.03255.x.

11. Monneret G, Gossez M, Venet F. Sepsis in PD-1 light. Crit Care 2016;20(1):1-2. DOI: 10.1186/s13054-016-1370-x.

12. De Pablo R, Monserrat J, Prieto A, Alvarez-Mon M. Role of circulating lymphocytes in patients with sepsis. Biomed Res Int 2014;2014:671087. DOI: 10.1155/2014/671087.

13. Walton AH, Muenzer JT, Rasche D, Boomer JS, Sato B, Brownstein BH, et al. Reactivation of multiple viruses in patients with sepsis. PLoS One 2014;9(6):1-13. DOI: 10.1371/journal.pone.0098819.

14. Kirby KA, Rubenfeld GD, Leisenring WM, Bulger EM, Neff MJ, Gibran NS, et al. Cytomegalovirus reactivation in critically ill immunocompetent patients. JAMA 2014;300(4):413-422. DOI: 10.1001/jama.300.4.413.

15. Tomino A, Tsuda M, Aoki R, Kajita Y, Hashiba M, Terajima T, et al. Increased PD-1 expression and altered T cell repertoire diversity predict mortality in patients with septic shock: a preliminary study. PLoS One 2017;12(1):1-12. DOI: 10.1371/journal.pone.0169653.

16. Heininger A, Haeberle H, Fischer I, Beck R, Riessen R, Rohde F, et al. Cytomegalovirus reactivation and associated outcome of critically ill patients with severe sepsis. Crit Care 2011;15(2):R77. DOI: 10.1186/ cc10069. Available from: http://ccforum.com/content/15/2/R77.

17. Vincent JL, Moreno R, Takala J, Willatts S, De Mendonça A, Bruining H, et al. The SOFA (Sepsis-related Organ Failure Assessment) score to describe organ dysfunction/failure. On behalf of the working group on sepsis-related problems of the European Society of Intensive Care Medicine. Intensive Care Med 1996;22(7):707-710. DOI: 10.1007/BF01709751. Available from: http://www.ncbi.nlm.nih gov/pubmed/8844239.

18. Kraft CS, Armstrong WS, Caliendo AM. Interpreting quantitative cytomegalovirus DNA testing: understanding the laboratory perspective. Clin Infect Dis 2012;54(12):1793-1797. DOI: 10.1093/cid/ cis212.

19. Hotchkiss RS, Monneret G, Payen D. Sepsis-induced immunosuppression: from cellular dysfunctions to immunotherapy. Nat Rev Immunol 2013;13(12):862-874. DOI: 10.1038/nri3552. Available from: http://www.ncbi.nlm.nih.gov/pubmed/24232462\%0A Available from: http://www.pubmedcentral.nih.gov/articlerender. fcgi?artid=PMC4077177.

20. Zhuang $Y$, Peng H, Chen Y, Zhou S, Chen Y. Dynamic monitoring of monocyte HLA-DR expression for the diagnosis, prognosis, and prediction of sepsis. 3. Sepsis and the immune response : reduced expression of HLA-DR. Front Biosci 2017;301:1344-1354. DOI: $10.2741 / 4547$.

21. Adrie C, Lugosi M, Sonneville R, Souweine B, Ruckly S, Cartier JC, et al. Persistent lymphopenia is a risk factor for ICU-acquired infections and for death in ICU patients with sustained hypotension at admission. Ann Intensive Care 2017;7(1):30. DOI: 10.1186/s13613-017-0242-0.

22. Drewry A, Samra N, Skrupky L, Fuller B, Compton S, Hotchkiss R. Persistent lymphopenia after diagnosis of sepsis predicts mortality. Shock 2014;42(5):383-391. DOI: 10.1097/SHK.0000000000000234.

23. Francisco LM, Sage PT, Sharpe AH. The PD-1 pathway in tolerance and autoimmunity. Immunol Rev 2010;236(1):219-242. DOI: 10.1111/j.1600065X.2010.00923.x.

24. La Rosa C, Krishnan A, Longmate J, Martinez J, Manchanda P, Lacey SF, et al. Programmed death-1 expression in liver transplant recipients as a prognostic indicator of cytomegalovirus disease. J Infect Dis 2008;197(1):25-33. DOI: 10.1086/523652.

25. Zhang Y, Li J, Lou J, Zhou Y, Bo L, Zhu J, et al. Upregulation of programmed death-1 on T cells and programmed death ligand-1 
on monocytes in septic shock patients. Crit Care 2011;15(1):1-9. DOI: 10.1186/cc10059.

26. Choi YJ, Kim SB, Kim JH, Park SH, Park MS, Kim JM, et al. Impaired polyfunctionality of CD8+ T cells in severe sepsis patients with human cytomegalovirus reactivation. Exp Mol Med 2017;49(9):1-8. DOI: 10.1038/emm.2017.146.

27. Schulte W, Bernhagen J, Bucala R. Cytokines in sepsis: potent immunoregulators and potential therapeutic targets-an updated view. Mediators Inflamm 2013;2013:165974. DOI: 10.1155/2013/ 165974.

28. Clement M, Humphreys IR. Cytokine-mediated induction and regulation of tissue damage during cytomegalovirus infection. Front Immunol 2019;10(1):1-9. DOI: 10.3389/fimmu.2019.00078.

29. Rojas JM, Avia M, Martín V, Sevilla N. IL-10: a multifunctional cytokine in viral infections. J Immunol Res 2017;2017:6104054. DOI: 10.1155/2017/6104054.

30. Silva TF, Concato VM, Tomiotto-Pellissier F, Gonçalves MD, Bortoleti BT da S, Tavares ER, et al. Reactivation of cytomegalovirus increases nitric oxide and IL-10 levels in sepsis and is associated with changes in renal parameters and worse clinical outcome. Sci Rep 2019;9(1):1-9. DOI: 10.1038/s41598-019-45390-x.

31. Matsumoto $\mathrm{H}$, Ogura $\mathrm{H}$, Shimizu $K$, Ikeda $M$, Hirose $T$, Matsuura $H$, et al. The clinical importance of a cytokine network in the acute phase of sepsis. Sci Rep 2018;8(1):1-4. DOI: 10.1038/s41598-01832275-8.

32. Remy S, Kolev-Descamps K, Gossez M, Venet F, Demaret J, Javouhey E, et al. Occurrence of marked sepsis-induced immunosuppression in pediatric septic shock: a pilot study. Ann Intensive Care 2018;8(1). DOI: 10.1186/s13613-018-0382-x.

33. Humar A, Louis PS, Mazzulli T, Mcgeer A, Lipton J, Messner $\mathrm{H}$, et al. Elevated serum cytokines are associated with cytomegalovirus infection and disease in bone marrow transplant recipients. J Infect Dis 1999;179(2):484-488. DOI: 10.1086/314602.

34. Reeves MB, Compton T. Inhibition of inflammatory interleukin-6 activity via extracellular signal-regulated kinase-mitogen-activated protein kinase signaling antagonizes human cytomegalovirus reactivation from dendritic cells. J Virol 2011;85(23):12750-12758. DOI: 10.1128/JVI.05878-11 